\title{
Estimation of K-distributed clutter by using characteristic function method
}

\begin{abstract}
Detection performance of the maritime radars is often limited by the unwanted sea echo or clutter. K-distribution is one of the long-tailed densities which is known in the signal processing community for fitting the radar sea clutter accurately. In this paper, a novel approach for estimating the parameter of K-distribution is presented. The method is derived from the empirical characteristic function of the quadrature components. Simulation results show a great improvement in term of estimated bias and variance, compared with any existing non-maximum likelihood method.
\end{abstract}

Keyword: Radar system; Sea clutter; Kï distribution; Parameter estimation; Characteristic function 